\title{
Ultrasound-guided bilateral transversus abdominis plane block versus spinal morphine for pain relief after caesarean section
}

\author{
Sofiene Ben Marzouk*, Laidi Bennasr, Imen cherni, Arbia Shili, Maha Touaibia, Hayen Maghrebi \\ University Hospital Assistant, Center of Maternity And Neonatology , La Rabta, Tunis, Tunisia
}

\begin{abstract}
Background: Ultrasound-guided transversus abdominis plane block is an effective method for pain relief after cesarean delivery. The gold standard to treat pain after cesarean delivery is spinal morphine administration; at the same time TAP is considered as an effective method to treat this pain. In this study, we compared efficiency and side effects of these techniques in patients undergoing elective cesarean delivery.

Methods: 104 women undergoing elective cesarean delivery under spinal anesthesia were randomized to receive either TAP block with Bupivacaine $0.25 \%$ (BUPICAÏNE, UNIMED, TUNISIA) or spinal morphine. All patients received a standard postoperative analgesia with Paracetamol intravenously. Rescue analgesia using Nefopam and morphine intravenously was given when necessary. Patients were assessed at 2, 4, 6, 12 and 24 hours after cesarean delivery. Visual analog scale (VAS) pain scores at rest and during the palpation of the uterine globe were recorded. Analgesic consumption, time to first analgesic request, heart rate, systolic and diastolic blood pressure were noted. Patients rated the severity of opoid side effects and their satisfaction with the protocol of analgesia.

Results: Pain scores at rest and during the palpation of the uterine globe during the first 24 postoperative hours were similar in both groups. The number of patients who received additional analgesia was similar in both groups. The intestinal transit recovery was earlier in the TAP block group with a statistically significant difference $(\mathrm{p}<10-3)$. Maternal satisfaction was similar in both groups.

Conclusion: TAP block and intrathecal morphine were of similar efficiency for pain relief after cesarean section. The incidence of side effects was comparable in both groups.
\end{abstract}

\section{Introduction}

Pain after caesarean section is severe during the first 48 postoperative hours, and then it gradually decreases during the second and the third day [1]. Post caesarean section pain management is an important clinical issue due to the increase of caesarean sections, to the need for an earlier perambulation and to an early recovery of motherchild relationship.

Common analgesic strategies are based on a multimodal approach combining spinal, epidural or systemic opioids to non-opioids such as Paracetamol, anti-inflammatory drugs, Tramadol or Nefopam. However, the use of opioids is usually associated with side effects such as nausea and vomiting, sedation, itch, urinary retention, delayed recovery of intestinal transit, respiratory depression and even hyperalgesia. Trying other analgesic techniques may be useful to reduce not only postoperative pain but also opioid-related side effects, and, as a result, to promote postoperative rehabilitation in order to limit the risk of thromboembolism on per partum [2]. The infiltration of the abdominal wall with local anesthetics is more commonly used for pain management. The transversus abdominis plane (TAP) block has recently been proposed to relieve the somatic pain component [3]. It is efficient especially when performed under ultrasound guidance. The aim of our study was to compare efficiency and side effects of spinal morphine to ultrasound-guided TAP block in patients undergoing elective caesarean delivery.

\section{Methods}

After local ethics committee agreement, we led a prospective randomized simple-blind study in the Maternity and Neonatology Center of Tunis. A clear explanation of the analgesic technique and pain assessment were given to the patients before obtaining their informed consent. Initially, we enrolled 104 ASA I or II parturients, all scheduled for elective cesarean delivery, via a Pfannenstiel incision under spinal anesthesia. Emergent cases, ASA III and IV, and nonconsenting parturients were not included. Parturients in whom spinal anesthesia failed were excluded $(n=0)$ as well as those in whom the typical biconvex lens image was not obtained while performing the ultrasound-guided TAP block $(n=2)$, those who had anesthetic or surgical complications $(n=2)$, and those who received any other analgesics than those prescribed in our study protocol $(n=0)$.

Randomization was performed using a computer-generated table of random numbers. Parturients were assigned to receive either

Correspondence to: Sofiene Ben Marzouk, Anesthesiologist, University Hospital Assistant, Center Of Maternity And Neonatology, La Rabta, Tunis, Tunisia, Tel: 0021699941968; E-mail: bmarzouksofiene@live.fr

Key words: anesthesia, cesarean delivery, intrathecal morphine, transversus abdominis plane block, ultrasonography

Received: March 08, 2016; Accepted: April 08, 2016; Published: April 12, 2016 
subarachnoid morphine (SM group) or TAP block (TAP group). Patients in the SM group received spinal anesthesia with $10 \mathrm{mg}$ of $0.5 \%$ hyperbaric bupivacaïne (BUPI, UNIMED, TUNISIA), $5 \mu \mathrm{g}$ of Sufentanil (SUFENTAMED, UNIMED, TUNISIA) and $100 \mu \mathrm{g}$ of preservative-free morphine (MORPHINE CHLOHYDRATE, AGUETTANT). Patients in the TAP group received spinal anesthesia with only $10 \mathrm{mg}$ of $0.5 \%$ hyperbaric bupivacaïne and $5 \mu \mathrm{g}$ of Sufentanil, they had in addition TAP block with $0.25 \%$ isobaric Bupivacaine $20 \mathrm{ml}$ on each side. The TAP block was performed at the end of surgery: A linear array 5-12 MHz ultrasound probe (MySono U6 $6^{\circ}$ MEDISON) was used. Imaging depth was set at 4 to $6 \mathrm{~cm}$. The probe was transversely oriented and placed at the midpoint between the lower costal margin and the iliac crest in the midaxillary line. The probe was angulated in order to identify the external oblique, internal oblique, and transversus abdominis muscles and their interfascial planes. A 20-gauge Quincke needle (adhe-els ${ }^{\star}$ ) was used, after scraping the tip in order to refine the sensation of loss of resistance when crossing the fascia. The needle was introduced via in plane technique into the neurofascial plane between the internal oblique and the transversus abdominis muscle, with the tip in the midaxillary line. A $3 \mathrm{~mL}$ test dose of the study solution was administered to check appropriate positioning. Twenty $\mathrm{mL}$ of $0.25 \%$ isobaric Bupivacaine was slowly injected on each side with negative aspiration every $5 \mathrm{~mL}$ under real-time ultrasounds visualization. Patients in both groups were given intravenous Paracetamol 1 gram every 6 hours. The first dose was given on arrival in postoperative care unit. Postoperative pain was assessed using a visual analogue scale (VAS). Breakthrough pain was treated with Nefopam (Acupan $\left.{ }^{\circledR}\right) 20 \mathrm{mg}$ every time the VAS score was more than $4 \mathrm{~cm}$. Intravenous morphine $5 \mathrm{mg}$ was given every time the VAS score remained above $4 \mathrm{~cm}$ after Nefopam infusion $[4,5]$.

The primary outcome of our study was pain scores at rest and during the palpation of uterine globe. Secondary outcomes were Nefopam and morphine consumption, time to first analgesic request, the occurrence of itch and postoperative nausea and vomiting, time to intestinal transit recovery and finally the maternal satisfaction. We aimed to detect a mean VAS score difference of $1 \pm 1.5 \mathrm{~cm}$ between the groups. A calculation based on $\alpha=0.05$ and a power of $90 \%$ yielded a sample size of 39 patients per group using a 2-tailed test. But we chose to enroll 52 parturients per group to improve the quality of our results.

Collected data were: age, weight, height, ASA status, parity, and number of deliveries, gestational age, and the indication for caesarean section. Patients were assessed for VAS scores at rest and during the palpation of uterine globe immediately after surgery then at the $2 \mathrm{nd}$, $3 \mathrm{rd}, 6$ th, 12th and 24th hour postoperative. We also noted heart rate, systolic and diastolic blood pressure, itch, postoperative nausea and vomiting, time to first analgesic request and the total given dose of Nefopam and morphine, and finally time to intestinal transit recovery. Patients were asked to rate their satisfaction by the mean of a threeitem scale (Excellent-Good-Bad).

Statistical analyses were performed using Statistical Package for the Social Sciences SPSS ${ }^{\oplus}$ version 20 software. Quantitative data were reported as mean \pm standard deviation (SD) and were analyzed using Student's $t$ test. Qualitative data were reported as numbers and percentages and were analyzed using Chi2 test. Statistical significance level was fixed at 0.05 .

\section{Results}

104 patients were enrolled in the study. Four patients were excluded: Two patients had post-partum hemorrhage requiring general anesthesia, two other patients were excluded because the typical biconvex lens image was not obtained while performing the ultrasound-guided TAP block. Consequently, only 100 patients were studied. Age, weight, height, body mass index, number of pregnancies, number of deliveries, gestational age, history of cesarean delivery, ASA status and duration of surgery were all similar in both groups (Table 1). VAS Pain scores at rest were similar in both groups during the first 24 hours postoperative (Table 2). VAS pain scores during palpation of the uterine globe were similar in both groups during the first 24 hours postoperative (Table 3). Breakthrough pain occurred in 18 patient (36\%) in TAP group versus 16 patients (32\%) in SM group

Table 1. Patients characteristics in both groups.

\begin{tabular}{|c|c|c|c|}
\hline Group & $\begin{array}{l}\text { TAP group } \\
(\mathbf{N}=50)\end{array}$ & $\begin{array}{c}\text { SM group } \\
(\mathbf{N}=\mathbf{5 0})\end{array}$ & $\mathbf{P}$ \\
\hline Age (year) & $32.3 \pm 4.3$ & $32.3 \pm 4.3$ & 0.55 \\
\hline Weight $(\mathrm{Kg})$ & $80.9 \pm 13.8$ & $78.1 \pm 11.7$ & 0.26 \\
\hline Height $(\mathrm{cm})$ & $162.9 \pm 5.6$ & $162 \pm 6.2$ & 0.43 \\
\hline BMI $\left(\mathrm{kg} / \mathrm{m}^{2}\right)$ & $30.5 \pm 4.9$ & $29.7 \pm$ & 0.38 \\
\hline Number of pregnancies & $2 \pm 1.2$ & $2 \pm 1.4$ & 0.87 \\
\hline Number of deliveries & $2.2 \pm 1$ & $2 \pm 1$ & 0.52 \\
\hline Gestational age (Week) & $38.7 \pm 1.1$ & $39.1 \pm 1.2$ & 0.08 \\
\hline History of cesarean delivery $(\%)$ & $72 \%$ & $62 \%$ & 0.39 \\
\hline ASA $1(\%)$ & $78 \%$ & $88 \%$ & \multirow{2}{*}{0.28} \\
\hline ASA $2(\%)$ & $22 \%$ & $12 \%$ & \\
\hline Duration of surgery (min) & $36.5 \pm 8.7$ & $35.9 \pm 6.6$ & 0.75 \\
\hline
\end{tabular}

Data are expressed as mean \pm SD or percentages

Table 2. VAS pain scores scale at rest.

\begin{tabular}{|c|c|c|c|}
\hline VAS (cm) & $\begin{array}{c}\text { TAP group } \\
\mathbf{( N = 5 0 )}\end{array}$ & $\begin{array}{c}\text { SM group } \\
\mathbf{( N = 5 0 )}\end{array}$ & P \\
\hline H2 & $1.0 \pm 1.5$ & $0.7 \pm 1.1$ & 0.19 \\
\hline H4 & $2.1 \pm 2.1$ & $1.9 \pm 1.9$ & 0.6 \\
\hline H6 & $2.8 \pm 2$ & $2.2 \pm 2.2$ & 0.12 \\
\hline H12 & $2.4 \pm 2.0$ & $2.3 \pm 1.8$ & 0.81 \\
\hline H24 & $2.1 \pm 1.4$ & $2.6 \pm 1.7$ & 0.1 \\
\hline
\end{tabular}

Data are expressed as mean $\pm \mathrm{SD}$

Table 3. VAS pain scores during palpation of the uterine globe.

\begin{tabular}{|c|c|c|c|}
\hline VAS (cm) & $\begin{array}{c}\text { TAP } \\
\mathbf{( N = 5 0 )}\end{array}$ & $\begin{array}{c}\text { MIT } \\
\mathbf{( N = 5 0 )}\end{array}$ & P \\
\hline H2 & $2.3 \pm 1.9$ & $2.3 \pm 2.1$ & 0.92 \\
\hline H4 & $3.7 \pm 2.4$ & $3.6 \pm 2.5$ & 0.92 \\
\hline H6 & $4.2 \pm 2.2$ & $4.0 \pm 2.6$ & 0.77 \\
\hline H12 & $4.4 \pm 2.4$ & $4.0 \pm 2.3$ & 0.43 \\
\hline H24 & $4.1 \pm 1.9$ & $4.5 \pm 2.1$ & 0.31 \\
\hline
\end{tabular}

Data are expressed as mean $\pm \mathrm{SD}$

Table 4. Evolution of systolic blood pressure and heart rate.

\begin{tabular}{|c|c|c|c|c|}
\hline \multirow{2}{*}{$\mathbf{H 2}$} & BP & $112 \pm 11$ & MIT $\mathbf{~}=\mathbf{5 0}$ & P \\
\hline \multirow{3}{*}{$\mathbf{H 4}$} & HR & $79 \pm 11$ & $113 \pm 13$ & 0.65 \\
\hline \multirow{2}{*}{$\mathbf{H 6}$} & BP & $112 \pm 10$ & $77 \pm 9$ & 0.08 \\
\cline { 2 - 5 } & HR & $80 \pm 10$ & $115 \pm 11$ & 0.15 \\
\hline \multirow{2}{*}{$\mathbf{H 1 2}$} & BP & $114 \pm 9$ & $79 \pm 10$ & 0.3 \\
\hline & HR & $81 \pm 9$ & $113 \pm 11$ & 0.72 \\
\hline \multirow{2}{*}{$\mathbf{H 2 4}$} & BP & $110 \pm 8$ & $80 \pm 11$ & 0.8 \\
\cline { 2 - 5 } & HR & $79 \pm 9$ & $113 \pm 8$ & 0.17 \\
\cline { 2 - 5 } & BP & $112 \pm 10$ & $114 \pm 7$ & 0.7 \\
\hline & HR & $78 \pm 8$ & $79 \pm 8$ & 0.2 \\
\hline
\end{tabular}

BP: Systolic blood pressure (mmHg); HR: Heart rate (bpm);Data are expressed as mean $\pm \mathrm{SD}$. 
$(\mathrm{p}=0.19)$ and was handled by Nefopam infusion. There was no need for Morphine injection in any patient. The average cumulative dose of Nefopam was similar in both groups $(26 \pm 10 \mathrm{mg}$ in TAP group $v s .32 \pm$ $20 \mathrm{mg}$ in SM group; $\mathrm{p}=0.4$ ). No difference was noted regarding time to first analgesic request in both groups ( 7 hours in TAP group vs. 9 hours in SM group; $\mathrm{p}=0.12$ ). Systolic blood pressure and heart rate were similar in both groups (Table 4). The incidence of itch was similar in both groups (76\% in TAP group vs. $78 \%$ in SM group; $\mathrm{p}=0.81$ ) as well as postoperative nausea and vomiting (18\% in TAP group vs. $28 \%$ in SM group; $\mathrm{p}=0.23) .45$ patients $(90 \%)$ in the TAP group restored their transit in the first 24 hours postoperative versus only 29 patients (58\%) in the SM group $\left(\mathrm{p}<10^{3}\right)$. Maternal satisfaction was similar for all items (excellent, good and bad) in both groups $(\mathrm{p}=0.94)$. in fact, satisfaction was excellent in $58 \%$ of cases in TAP group vs. $60 \%$ in SM group.

\section{Discussion}

Ultrasound-guided blockade of the abdominal wall was found to be efficient for pain relief after cesarean delivery [6,7]. It is associated with a decrease of other analgesic consumption such as Tramadol $[7,8]$ or morphine [9], and with a longer time to first analgesic request [9].

Studies have compared the analgesic efficacy of spinal morphine administration to TAP block after cesarean section. Our study found that spinal morphine and TAP block provided a similar analgesic efficiency. Kanazi et al. [8] however, did not find a difference in VAS pain scores with $0.2 \mathrm{mg}$ of spinal morphine. But, using an increasing dose of spinal morphine (>0.1 mg) makes no analgesic benefit but many side effects [10-12]. Actually, the optimal dose of spinal morphine is thought to be of only $75 \mu \mathrm{g}$ [13]. As found in our study, Loane et al. [14] reported no difference in pain scores while comparing $100 \mu \mathrm{g}$ of spinal morphine to ultrasound-guided TAP block with $100 \mathrm{mg}$ of Ropivaciane in patients undergoing elective cesarean section. Several combinations of spinal morphine to TAP block were reported [1518], they used either Bupivacaine or Ropivacaine and globally found that the association of spinal morphine with TAP block may improve analgesia and even delay the first analgesic request but it is associated with an increasing incidence of side effects. Spinal morphine, but not TAP block, is thought to improve pain scores and morphine consumption [18]. The association of TAP block with spinal morphine may not give additional analgesic gain [18]. This fact may be due to the absence of ultrasound guidance when performing the TAP block. Indeed, ultrasound-guided nerve blocks offers the advantage of real time imaging of the needle trajectory and local anesthetics spread, which may improve both safety and block effectiveness [19].

In our study, total doses of rescue analgesia were comparable in both groups. Kanazi et al. [8] showed reduced total tramadol doses during the first 12 hours in the spinal morphine group; however, they used higher doses $(0.2 \mathrm{mg})$ of spinal morphine.

In our study, the time to first analgesic request was similar in both groups. Kanazi et al. [8] reported a longer time to first analgesic request in the spinal morphine administration group ( 8 hours vs 4 hours).

The mean heart rate and blood pressure were similar in both groups. We did not find studies that use these data for assessment of pain relief.

Spinal morphine used to be the " gold standard " technique for post cesarean pain relief [20-22]. The recommended dose of 100 $\mu \mathrm{g}$, is in fact a good compromise between efficiency and side effects (respiratory depression, itch, nausea, vomiting and delay recovery of transit) [23]. The incidence of itch, nausea and vomiting was comparable in both groups in our study. The restoration of transit was significantly delayed in the spinal morphine group compared to the TAP block group. Nausea and vomiting are the commonest side effects of spinal morphine; it is seen in more than $30 \%$ of cases [9-14,18-24]. A meta-analysis [15] including nine studies found that incidence of nausea is reduced during the first 12 postoperative hours with TAP block analgesia. However, none of these studies mentioned the effect of the analgesic protocols on the recovery time of transit.

Our study as well as, Kanazi's study [8], found that spinal morphine and TAP block provided equivalent maternal satisfaction. The conjunction of spinal morphine administration with the TAP block also improved maternal satisfaction [17].

Because of its analgesic benefit, the TAP block may be useful as rescue analgesia rather than a routine technique [25]. Ultrasoundguided TAP block seems to be safe but cases of visceral injury were reported [14,26-28]. Injection of high local anesthetics volumes may increase the risk of systemic toxicity [29]. On the other side, the optimal dose of local anesthesia as well as the duration of analgesia remains poorly estimated. Carney et al. [30] founded TAP block to be efficient for up to 48 hours.

Despite their well-established efficiency, Non-steroidal antiinflammatory drugs [31] were not used in our study in order to bring out the role of local anesthetics. In our study, we did not assess the success rate of sensory distribution of the block, because residual sensory block related to spinal anesthesia may remain in the early postoperative period. We did not measure the Bupivacaine blood rates in the TAP group.

\section{Conclusion}

TAP block and spinal morphine have similar efficiency for pain relief after cesarean section. No difference was noted regarding the VAS pain scores, the total cumulative dose, the time to first analgesic request, the systolic blood pressure and heart rate. The restoration of transit was significantly delayed in the spinal morphine group compared to the TAP block group. The incidence of itch, postoperative nausea, vomiting and maternal satisfaction was comparable in both groups.

\section{References}

1. French Society of Anesthesia and Intensive Care (1998) Jury recommendations. Management of postoperative pain in adults and children. Consensus conference. Ann Fr Anesth-Réanim 17: 445-461.

2. Keita-Meyer H (2006) Postoperative analgesia after caesarean section. Consensus Conference. 677-688.

3. Bloc S, Bouaziz H (2010) Transversus Abdominis Plane Block. 52nd National Congress of Anesthesia and resuscitation. Conferences Update Sfar.

4. Hebbard P, Royse C (2008) Audit of transverse abdominus plane block for analgesia following caesarean section. Anaesthesia 63: 1382. [Crossref]

5. El-Dawlalty AA, Tirkistani A, Kettner SC, Machata AM, Delvi MB, et al. (2009) Ultrasound-guided transversus abdominis plane block: description of a new technique and comparison with conventional systemic analgesia during laparoscopic cholecystectomy. BR J Anaesth 102: 763-767. [Crossref]

6. McDonnell JG, Curley G, Carney J, Benton A, Costello J, et al. (2008) The analgesic efficacy of transversus abdominis plane block after cesarean delivery: A randomized controlled trial. Anesth Analg 106: 186-191. [Crossref]

7. Eslamian L, Jalili Z, Jamal A, Marsoosi V, Movafegh A (2012) Transversus abdominis plane block reduces postoperative pain intensity and analgesic consumption in elective cesarean delivery under general anesthesia. J Anesth 26: 334-338. [Crossref]

8. Kanazi GE, Aouad MT, Abdallah FW, Khatib MI, Adham AM, et al. (2010) The 
Analgesic Efficacy of Subarchnoid Morphine in comparison with UltrasoundGuided Transversus Abdominis Plane Block After Cesarean Delivery: A Randomized Controlled Trial. Society for Obstetric Anesthesia and Perinatalogy. Anesth Analg 111: 475-481. [Crossref]

9. Belavy D, Cowlishaw PJ, Howes M, Phillips F (2009) Ultrasound-guided transversus abdominis plane block for analgesia after Caesarean delivery. Br J Anaesth 103: 726730. [Crossref]

10. Uchiyama A, Nakano S, Ueyama H, Nishimura M, Tashiro C (1994) Low dose intrathecal morphine and pain relief following caesarean section. Int J Obstet Anesth 3: 87-91. [Crossref]

11. Milner AR, Bogod DG, Harwood RJ (1996) Intrathecal administration of morphine for elective Caesarean section. A comparison between $0.1 \mathrm{mg}$ and $0.2 \mathrm{mg}$. Anaesthesia 51: 871-873. [Crossref]

12. Girgin NK, Gurbet A, Turker G, Aksu H, Gulhan N (2008) Intrathecal morphine in anesthesia for cesarean delivery: dose-response relationship for combinations of lowdose intrathecal morphine and spinal bupivacaine. J Clin Anesth 20: 180-185. [Crossref]

13. Palmer CM, Emerson S, Volgoropolous D, Alves D (1999) Dose-response relationship of intrathecal morphine for postcesarean analgesia. Anesthesiology 90: 437-444. [Crossref]

14. Loane H, Preston R, Douglas MJ, Massey S, Papsdorf M, et al. (2012) A randomized controlled trial comparing intrathecal morphine with transversus abdominis plane block for post-cesarean delivery analgesia. International Journal of Obstetric Anesthesia 21 : 112-118. [Crossref]

15. Mishriky BM1, George RB, Habib AS (2012) Transversus abdominis plane block for analgesia after cesarean delivery: a systematic review and meta-analysis. Can J Anesth 59: 766-778. [Crossref]

16. Anthony Ghosn, Jean Claude Stephan, Neematallah Aghnatios et al. (2012) Superiority of the Transverse abdominal plane Association (TAP) block and intrathecal morphine compared with the MIT alone in the treatment of post cesarean pain. Can J Anaesth 59: 766-768.

17. Lee AJ, Palte HD, Chehade JM, Arheart KL, Ranasinghe JS, et al. (2013) Ultrasoundguided bilateral transversus abdominis plane blocks in conjunction with intrathecal morphine for postcesarean analgesia. $J$ Clin Anesth 25: 475-482. [Crossref]

18. McMorrow RC, Ni Mhuircheartaigh RJ, Ahmed KA, Aslani A, Ng SC, et al. (2011) Comparison of transversus abdominis plane block vs spinal morphine for pain relief after Caesarean section. Br J Anaesth 106: 706-712. [Crossref]
19. Barrington MJ, Ivanusic JJ, Rozen WM, Hebbard P (2009) Spread of injectate after ultrasound-guided sub costal transversus abdominis plane block: a cadaveric study. Anesthesia 64: 745-750. [Crossref]

20. Dahl JB, Jeppesen IS, Jorgensen H, Wetterslev J, Møiniche S (1999) Intraoperative and postoperative analgesic efficacy and adverse effects of intrathecal opioids in patients undergoing cesarean section with spinal anesthesia: A qualitative and quantitative systematic review of randomized controlled trials. Anesthesiology 91: 1919-1927. [Crossref]

21. Scott PV, Bowen FE, Cartwright P, Rao BC, Deeley D, et al. (1980) Intrathecal morphine as sole analgesic during labour. $\mathrm{Br}$ Med $J$ 281: 351-353. [Crossref]

22. Tong C, Conklin D, Eisenach JC (2006) A pain model after gynecologic surgery: the effect of intrathecal and systemic morphine. Anesth Analg 103: 1288-1293. [Crossref]

23. Wong JY, Carvalho B, Riley ET (2013) Inrathecal morphine 100 and $200 \mu \mathrm{g}$ for postcesarean delivery analgesia: A trade-off between analgesic efficacy and side effects. International Journal of Obstetric Anesthesia 22: 36-41. [Crossref]

24. Chaney MA (1995) Side effects of intrathecal and epidural opioids. Can J Anaesth 42 891-903. [Crossref]

25. Mizra F, Carvalho B (2013) Transversus abdominis plane blocks for rescue analgesia following Cesarean delivery: A case series. Can J Anaesth 60: 299-303. [Crossref]

26. Frigon C, Mai R, Valois-Gomez T, Desparmet J (2006) Bowel hematoma following an iliohypogastric-ilioinguinal nerve block. Pediatric Anaesth 16: 993-996. [Crossref]

27. Lancaster P, Chadwick M (2010) Liver trauma secondary to ultrasound-guided transversus abdominis plane block. Br J Anaesth 104: 509-510. [Crossref]

28. Weintraud M, Marhofer P, Bosenberg A, Kapral S, Willschke H, et al. (2008) Ilioinguinal/iliohypogastric blocks in children: where do we administer the local anesthetic without direct visualization. Anesth Analg 106: 89-93. [Crossref]

29. Griffiths JD, Barron FA, Grant S, Bjorksten AR, Hebbard P (2010) Plasma ropivacaine concentration after ultrasound-guided transversus abdominis plane block. Br J Anaesth 105: 853-856. [Crossref]

30. Carney J, McDonnell JG, Ochana A, Bhinder R, Laffey JG (2008) The transversus abdominis plane block provides effective postoperative analgesia in patients undergoing total abdominal hysterectomy. Anesth Analg 107: 2056-2060. [Crossref]

31. Abdallah FW, Halpern SH, Margarido CB (2012) Transversus abdominis plane block for postoperative analgesia after Caesarean delivery performed under spinal anaesthesia? A systematic review and meta-analysis. Br J Anaesth 109: 679-687. [Crossref]

Copyright: C2016 Marzouk SB. This is an open-access article distributed under the terms of the Creative Commons Attribution License, which permits unrestricted use, distribution, and reproduction in any medium, provided the original author and source are credited. 